\title{
Development of automatic body condition scoring using a low-cost 3-dimensional Kinect camera
}

\author{
Roii Spoliansky, ${ }^{*}$ Yael Edan, ${ }^{*}$ Yisrael Parmet, ${ }^{*}$ and Ilan Halachmi* ${ }^{1}$ \\ *Department of Industrial Engineering and Management, Faculty of Engineering Sciences, Ben-Gurion University of the Negev, \\ Beer-Sheva 84105, Israel \\ †The Institute of Agricultural Engineering, Agricultural Research Organization (ARO), The Volcani Center, PO Box 6, Bet Dagan 50250, Israel
}

\begin{abstract}
Body condition scoring (BCS) is a farm-management tool for estimating dairy cows' energy reserves. Today, BCS is performed manually by experts. This paper presents a 3-dimensional algorithm that provides a topographical understanding of the cow's body to estimate BCS. An automatic BCS system consisting of a Kinect camera (Microsoft Corp., Redmond, WA) triggered by a passive infrared motion detector was designed and implemented. Image processing and regression algorithms were developed and included the following steps: (1) image restoration, the removal of noise; (2) object recognition and separation, identification and separation of the cows; (3) movie and image selection, selection of movies and frames that include the relevant data; (4) image rotation, alignment of the cow parallel to the x-axis; and (5) image cropping and normalization, removal of irrelevant data, setting the image size to $150 \times 200$ pixels, and normalizing image values. All steps were performed automatically, including image selection and classification. Fourteen individual features per cow, derived from the cows' topography, were automatically extracted from the movies and from the farm's herd-management records. These features appear to be measurable in a commercial farm. Manual BCS was performed by a trained expert and compared with the output of the training set. A regression model was developed, correlating the features with the manual BCS references. Data were acquired for $4 \mathrm{~d}$, resulting in a database of 422 movies of 101 cows. Movies containing cows' back ends were automatically selected (389 movies). The data were divided into a training set of 81 cows and a test set of 20 cows; both sets included the identical full range of BCS classes. Accuracy tests gave a mean absolute error of 0.26 , median absolute error of 0.19 , and coefficient of determination of 0.75 ,
\end{abstract}

Received November 7, 2015.

Accepted May 1, 2016.

${ }^{1}$ Corresponding author: halachmi@volcani.agri.gov.il with $100 \%$ correct classification within 1 step and $91 \%$ correct classification within a half step for BCS classes. Results indicated good repeatability, with all standard deviations under 0.33 . The algorithm is independent of the background and requires 10 cows for training with approximately 30 movies of $4 \mathrm{~s}$ each.

Key words: sensor, cattle, computer vision, body condition scoring, precision livestock farming

\section{INTRODUCTION}

Automatic and objective BCS will help to ensure that the cow is in the correct condition for each stage of her annual cycle and that appropriate dietary changes can be made to correct any deficiencies. Achieving correct body condition at calving is important to avoid calving difficulties and losses. When meeting the extra nutritional demands of high-yielding cows, body condition is a vital indicator of excessive weight loss in early lactation, which can lead to metabolic disorders and should be avoided. Condition scoring is particularly useful as an aid to dry cow and precalving management. The objective is to ensure that cows calve down safely to prepare cows for early lactation. Fat cows may develop fatty liver disease or ketosis and are more prone to milk fever, mastitis, lameness, and infertility. At service, dairy cows should not be in energy deficit by this stage, as this may result in low fertility.

Body condition scoring is a management tool in dairy farming (Edmondson et al., 1989; Ferguson, 1996; Bewley et al., 2008; Halachmi et al., 2008). Changes in cows' BCS value have been found to be a good forecasting tool for problems with cow health, production, and reproduction (Wildman et al., 1982). A cow's energy reserves are evaluated by BCS, on a 5-point scale, where 1 is an emaciated cow and 5 is obese (Ferguson et al., 1994; Ferguson, 1996). Today, BCS is performed manually by trained experts using tactile (feeling the fat and bone tissue of the cow) or visual (looking at the shape of the cow) methods (Hady et al., 1994). Manually performed BCS requires experienced person- 
nel; it is time-consuming, provides a subjective estimation, and cannot be automatically collaborated with computerized herd-management software (Coffey et al., 2003; Ferguson et al., 2006; Halachmi et al., 2008).

Several attempts have been made to automate the BCS process, mainly by including an analysis of the cow's contour and shape (Sharony, 2003; Peacock et al., 2006; Halachmi et al., 2008; Bercovich et al., 2013; Salau et al., 2014; Tedín et al., 2014; Weber et al., 2014; Caccamo et al., 2015). However, these systems still require some level of human interference. Recently, one commercial application has appeared (DeLaval, 2015), but its accuracy was not published in a scientific publication, the cow needs to stand, and it is not based on a low-cost camera.

Three-dimensional (3D) computer vision has advanced in the last few years due to recently acquired ability to handle large data sets (Shirai, 2012), a better understanding of the curvatures in the picture, and background issues (Cyganek and Siebert, 2011); 3D computer vision is also emerging in precision livestock farming (Salau et al., 2014; Viazzi et al., 2014; Weber et al., 2014; Shelley et al., 2015). Nevertheless, low-cost $3 \mathrm{D}$ computer vision has not yet been applied to the problem of automatic BCS. This research focused on achieving a proof-of-concept for the development of a low-cost 3D computer vision system, with no human included in the BCS process, aimed at providing automatically acquired, objective sensing, and accurate BCS values with no dependence on image background.

\section{MATERIALS AND METHODS}

\section{System Setup}

A walk-through system was built at the exit of the milking parlor. The images were captured while the cows walked voluntarily below the camera, eliminating the need to stop the cows or interfere with cow traffic. A Microsoft Kinect camera (version 1; Microsoft Corp., Redmond, WA) was placed $2.5 \mathrm{~m}$ above the concrete platform. The images were captured automatically using a passive infrared motion detector which served as the camera trigger. The trigger was placed $0.3 \mathrm{~m}$ before the camera as the cows exited the milking parlor.

The camera and trigger were connected to a personal computer and operated using OpenNI software (OpenNI 1.6, PrimeSense, Tel-Aviv, Israel). Each time the camera was triggered, a 4-s movie clip was made containing between 100 and 120 frames (images in the movie). The setup was installed at the ARO Research Dairy Farm in Bet Dagan, Israel. Data were collected between October 30 and November 3, 2013.

\section{Data Acquisition}

Automatic sampling was run continuously for 4 consecutive days and nights without any human interference. All movies, with depth images, were automatically saved, dated, and time-coded. The movies were automatically split into singulated $480 \times 640$ pixel images using Matlab (The MathWorks Inc., Natick, MA). Each image contained the distance between the sensor and the floor $(2.5 \mathrm{~m})$ and the distance to the cow, which depended on cow height. All of the image processing steps were performed automatically. Additional data-cow age and weight (from last weighing up to a week before the movie capture) - were taken from the farm records. A trained expert evaluated BCS manually on the first day of data collection. The trained expert provided a gold reference. A credible gold reference is an issue in every new sensor development. A previous study (Halachmi et al., 2008) applied ultrasound measurements; however, ultrasound appeared to be biased according to the ultrasound image interpreter (Halachmi et al., 2008). Later, Halachmi et al. (2013) averaged values of 2 experts, but differences among the observers were large, leading to criticism. Therefore, the validity of the expert was not questioned in this research (the expert is well known for his accuracy and has years of experience in BCS, including his entire postdoctoral period which was focused on BCS). The raw data included 101 cows. Filtering out frames that lacked the cows' back ends or had obstacles interfering with the view of the cow, as well as empty movies resulted in 14,474 frames for the database analysis; all 101 cows were represented in the database.

Each movie was originally composed of about 100 images; relevant images were selected at the imageselection stage. The cows in the research were randomly selected to ensure that all BCS, ages, and stages of lactation are included in the sample. Data were paired according to BCS classes (i.e., scores of 1 to 5) and randomly split into training and testing data sets, with each set consisting of different cows. The training set and the testing set had cows from all BCS classes, with similar distribution relative to each other as well as to the total herd. The regression parameters were calibrated on the training database, then the model was applied on the testing database with the gold standard being the BCS values from the human expert.

\section{Feature Selection and Extraction}

Initially, potential features were selected by examining the way the expert was evaluating the BCS. Then, the suspected features were normalized to between 0 
Table 1. Investigated features (X1-X14) and their correlation to BCS (Y)

\begin{tabular}{lll}
\hline Features & Meaning & Correlation to BCS \\
\hline Y & Cow BCS & Relative height of the right edge of the cow's back end \\
X1 & Relative height of the cow's spine & 0.13 \\
X2 & Relative height of the front left side of the cow's back end & 0.11 \\
X3 & Relative height of front right side of the cow's back end & 0.38 \\
X4 & Relative height of the back left side of the cow's back end (next to the tail) & 0.46 \\
X5 & Relative height of back right side of the cow's back end (next to the tail) & 0.46 \\
X6 & Average relative height of the cow's back end & 0.46 \\
X7 & Standard deviation of relative height of the cow's back end & 0.33 \\
X8 & Height of the cow & 0.18 \\
X9 & Number of minimum height points on the cow's back end & 0.02 \\
X10 & Number of maximum height points on the cow's back end & 0.15 \\
X11 & Cow's weight & 0.09 \\
X12 & Cow's age & 0.32 \\
X13 & Average distance from the cow's contour to the center of the cow's right edge & 0.09 \\
X14 & &
\end{tabular}

and 1 to obtain the same scale for all cows and all features. Subsequently, features selection was based on statistical contribution $\left(\mathrm{R}^{2}\right.$, cross-correlation coefficients, step-wise regression, and so on) of each feature. The means of small groups of pixel values, $\mathrm{X} 1$ to $\mathrm{X} 6$ (Table 1), were selected using correlations of the pixels (relative height) to BCS. The features were selected by finding the pixel with the largest correlation and the 9 neighboring pixels (also neighbors of selected neighbors) with the largest correlation to the BCS. The process was repeated 6 times to obtain 6 local features (the number of maximum and minimum points in the correlation image).

The set of features calculated from the output of the image-processing algorithm, X7 to X11 and X14 (Table 1 ), were (1) number of local minima and maxima in an image that correlate to the number of bulges in the cow's back; (2) the mean value of the image (i.e., the mean value of the relative heights that correlate to the degree of change from the highest pixel); (3) the standard deviation of the image that correlates to the degree of change from the mean value; and (4) the average distance between the contour and the middle of the right column that correlates to the shape of the back part of the contour. Prior data consisted of the cow's age (X13) and last recorded weight (X12). These were obtained from the herd-management software.

\section{Prediction Models}

The models were trained on the training data and tested with the testing data by comparing the automatic BCS to the expert's manual BCS using several methods. Polynomial regression models where determined in a-priori analysis with all potential variables up to a quadratic equation. During the stepwise regression and the cross-correlation coefficient analysis (Matlab's func- tion corrcoef), it seemed that the variables representing the cow's back end height next to the cow tail (left 5 times and right 6 times) are highly correlated with each other and one can be excluded from the model.

\section{Model Performance Evaluation and Validation}

Regression model evaluation was based on measures of model accuracy including the mean absolute error (MAE) and median absolute error, the correlation $\left(\mathrm{R}^{2}\right)$ between the model results and the BCS target values, the percentage of correct classifications, and the percentage of classifications that were less than or exactly one-quarter, one-half, three-quarters, and 1 class away from the target BCS class.

\section{Sensitivity Analyses}

The following sensitivity analyses were performed:

- K-fold cross-validation of the training sets (with $\mathrm{K}$ values of 10,20 , and 30 );

- size of the training set (10 random selections of part of the training set performed for each of the following training set sizes: $90,80,70,60,50,40$, $30,20,10,5$, and $3 \%$ );

- type and distribution of the training data, where 100 movies with distractions were inserted into the database to ensure that the algorithm can delete movies (with false data considered to be cows no back ends in them, dairy farmer passing through, more than 1 cow in the movie, cows touching, movie with no cows, and movie with cow head looking from behind the fence); and

- noise, including random Gaussian noise, with several ratio-to-signal values (200:1, 100:1, 90:1, $80: 1,70: 1,60: 1,50: 1,40: 1$, and 20:1) added to the 
features before entering them into the prediction models and the results were tested.

\section{Image Processing}

Workflow. Image processing normalized images that included the cow's back end to equal size and rotation. Images without the cow's back end or with obstacles (e.g., humans or dogs passing below the camera, other cows hiding in part of the cow image) were filtered out automatically during the image-processing algorithm. Image processing included the following steps: restoration, object recognition and separation, image rotation, and image cropping and normalization. The Matlab software code was written by the first author specifically for this study to meet research needs. Figure 1 shows the workflow of the image processing steps. The workflow was automatic, without any human intervention.
Image Restoration. A linear interpolation based on neighboring pixels and a neighbor image was applied to repair small holes in the images. The holes were caused by sunlight, moonlight, and rapid movements by the cows. The restoration was performed in 2 steps: (1) if the left neighbor pixel in the earlier frame had a value, the pixel received that value, and (2) missing pixels received the value of the linear interpolation of 2 neighboring pixels (i.e., the value of the closest neighbor pixel with a preference for the one on the right side; cows were walking from left to right). Figure 2 shows an example of holes in the image (encircled). Figure 3 shows 2 images: on the left noise is shown as dark blue spots and on the right is the image after restoration.

Object Recognition and Separation. Two thresholds were used to separate the main object from the background. In threshold 1, a preset distance from the camera was determined empirically to be $1,800 \mathrm{~mm}$. A value that was further away from the camera was
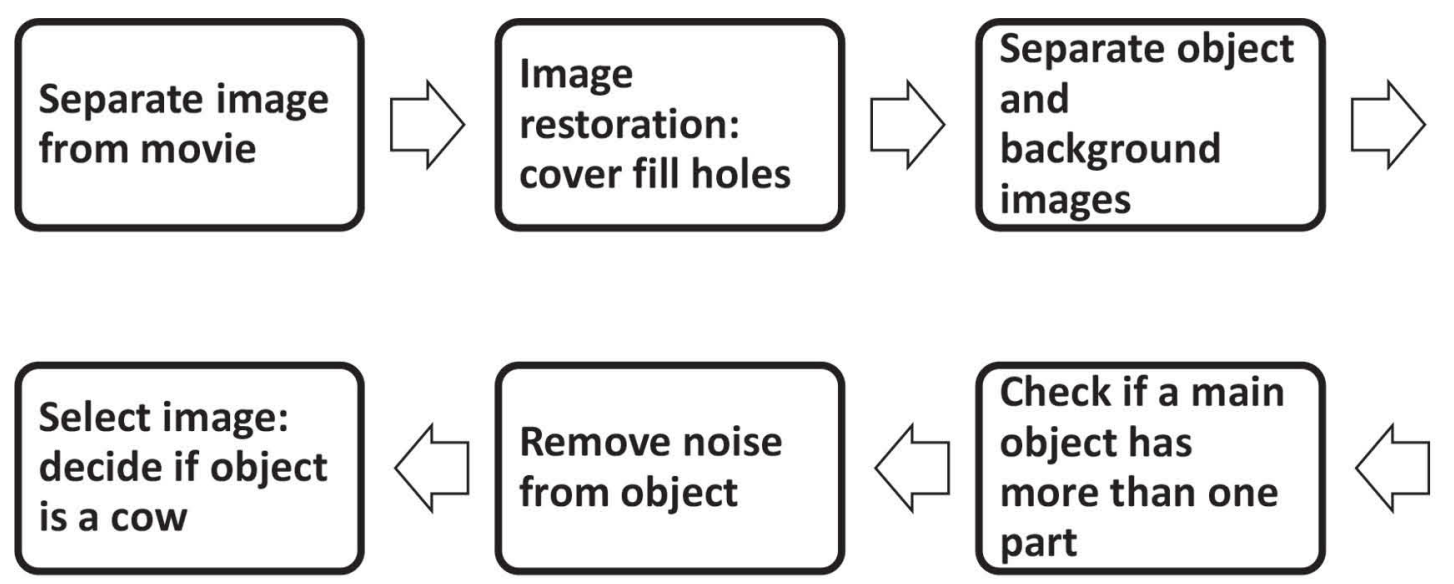

Segmentation:
remove
secondary
objects

Subtract one image from the other

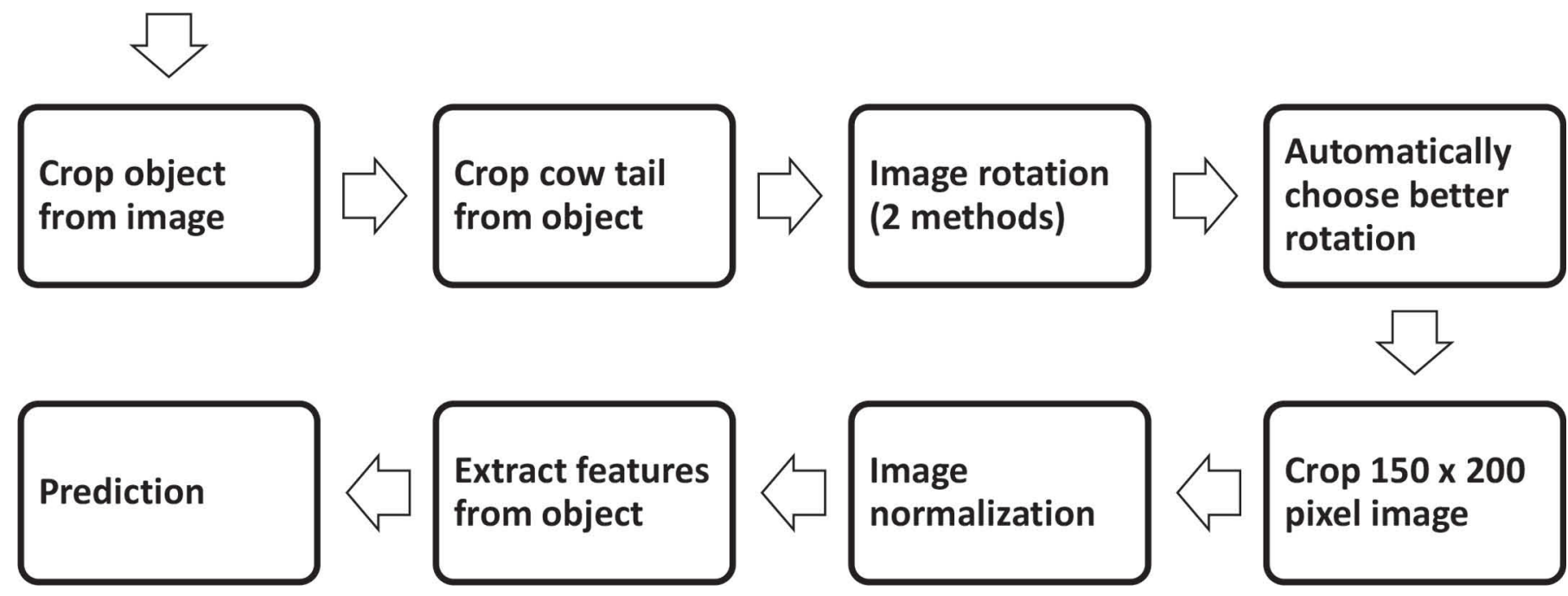

Figure 1. Workflow of image processing and prediction algorithms. 


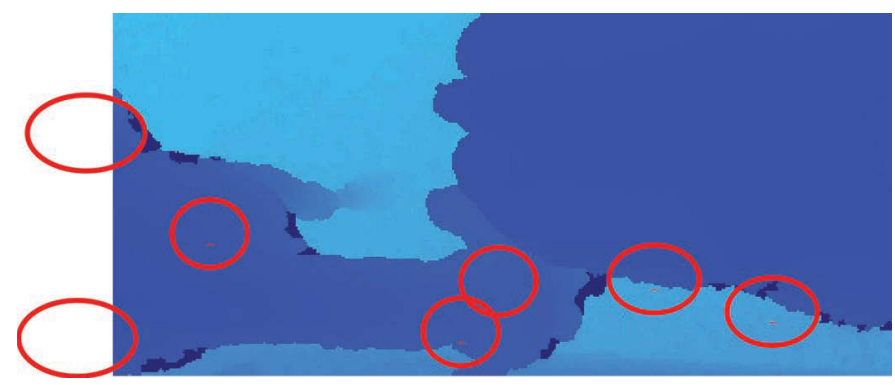

Figure 2. Examples of holes in the image. Color version available online.

ignored. This threshold overcame the dependency on image background. Threshold 2 was a dynamic threshold, automatically calculated for each image separately using the method of Otsu (1975) to select the main object in the image. The last frame before a cow entered the camera field of view was set as the background image. The background was subtracted from the successive images to consider only the objects that do not exist in the background image.

A third threshold was used to detect a cow: objects were separated by connectivity analysis (with 8 neighbors) and the size of each object was measured by counting the number of pixels in it. The largest object was selected (cow) and all other objects were deleted from the image. To detect 2 cows that were recognized as 1 object, we used convolutions and tested whether the derivative value, obtained from the convolution, was in the range of the selected thresholds (larger than 90 and smaller than 500). Table 2 shows the convolution filter values: this filter detected the contour of the object, and after the filter was used on the image each pixel that was under 1.8 and above 1 was selected. Table 2 also reports the filter that smoothed the contour. The

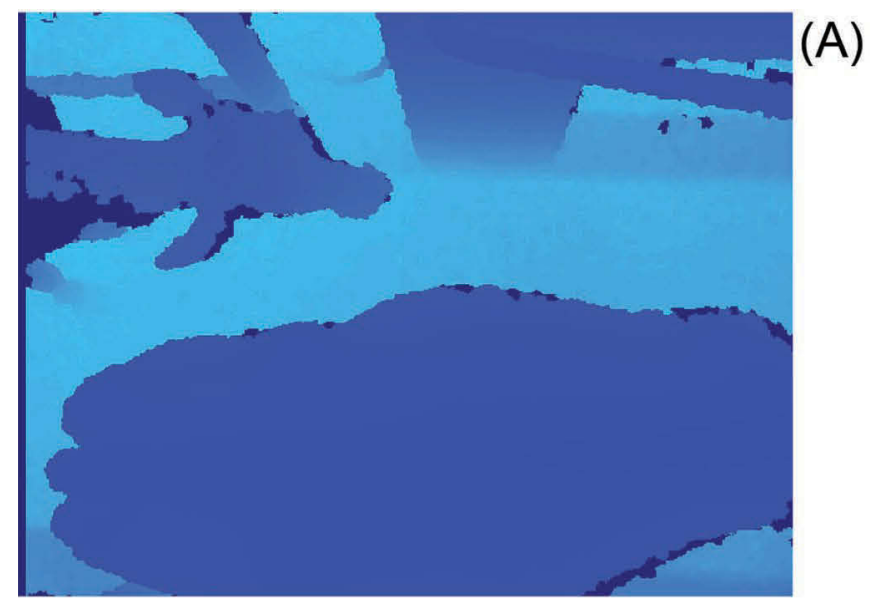

Table 2. Filters used for the object extraction ${ }^{1}$

\begin{tabular}{cllrrrrrr}
\hline $\begin{array}{l}\text { Convolution filter } \\
\text { values }\end{array}$ & \multicolumn{4}{c}{$\begin{array}{c}\text { Contour } \\
\text { smoothing filter }\end{array}$} & \multicolumn{3}{c}{ Averaging filter } \\
\hline 0.1 & 0.1 & 0.1 & 0 & 1 & 0 & 0.1 & 0.1 & 0.1 \\
0.1 & 1 & 0.1 & 1 & 10 & 1 & 0.1 & 0.2 & 0.1 \\
0.1 & 0.1 & 0.1 & 0 & 1 & 0 & 0.1 & 0.1 & 0.1 \\
\hline
\end{tabular}

${ }^{1}$ Numbers in table are coefficients without units.

averaging filter in Table 2 covered the single-pixel holes in the contour by detecting pixel values of less than 0.5 and setting their values to the value of a dynamic $3 \times 3$.

Image Selection. The following features were applied to ensure that the image was of a single cow: (1) the width of the object should be smaller than a matrix row index of 320; (2) the size of the object, calculated as the number of pixels, should be larger than 60,000 ; $(3,4)$ binary values representing the presence of an object in the first and last column of the image were used to ensure that the cow's back end is in the image, the first column's binary value should be 0 (the back end has already entered the frame) and the last column's binary value should be 1 (for the cow's length, if the back end is in the frame the head must be out of it); (5) the maximum height of the object was used to make sure that the object is a cow and not a fence or a dairy farmer, the minimum distance from the camera should be larger than 1,100 mm; (6) the width of the object's end (in the first column of the object) was compared with the width of the object's middle (in the middle column of the object) to ensure that the object's shape is similar to a cow's shape (i.e., narrower at the back and broader in the middle).

Image Cropping. All parts of the image with no object in them were cropped. Image columns and rows

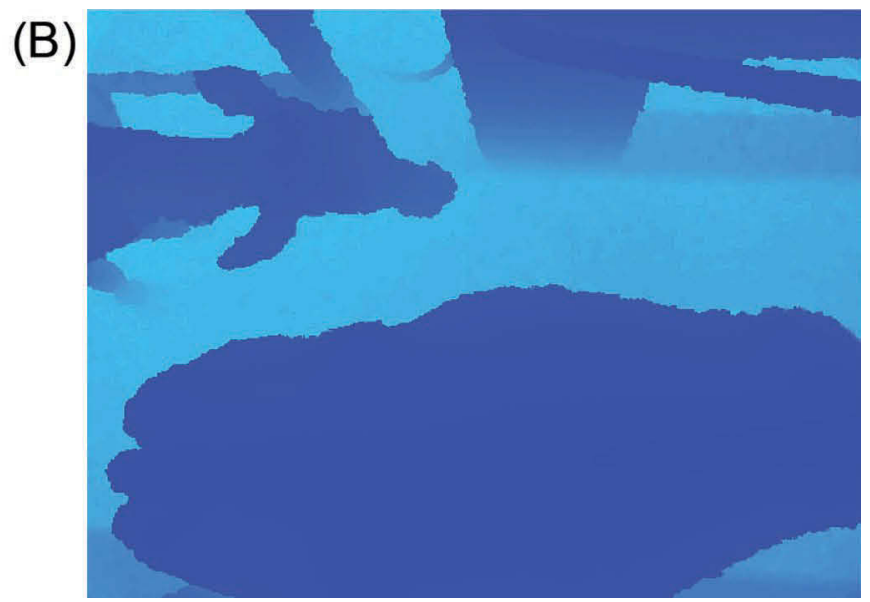

Figure 3. Image before (A) and after (B) restoration. Color version available online. 


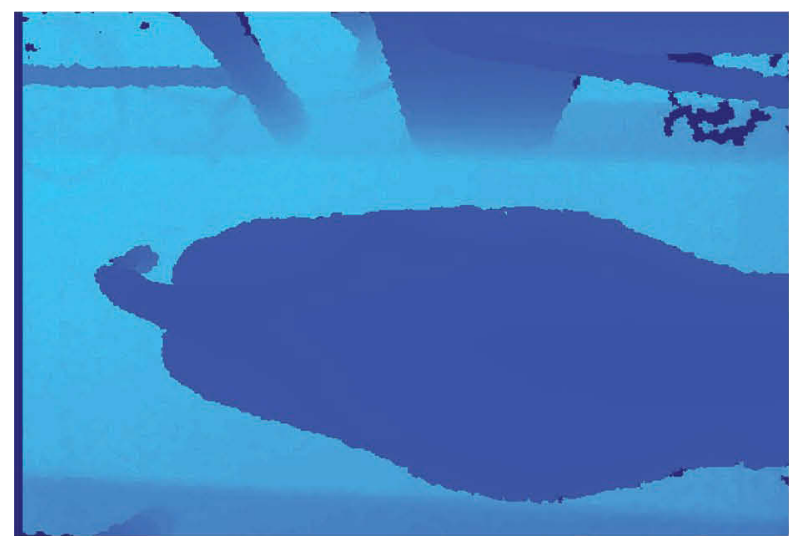

(A)

(B)

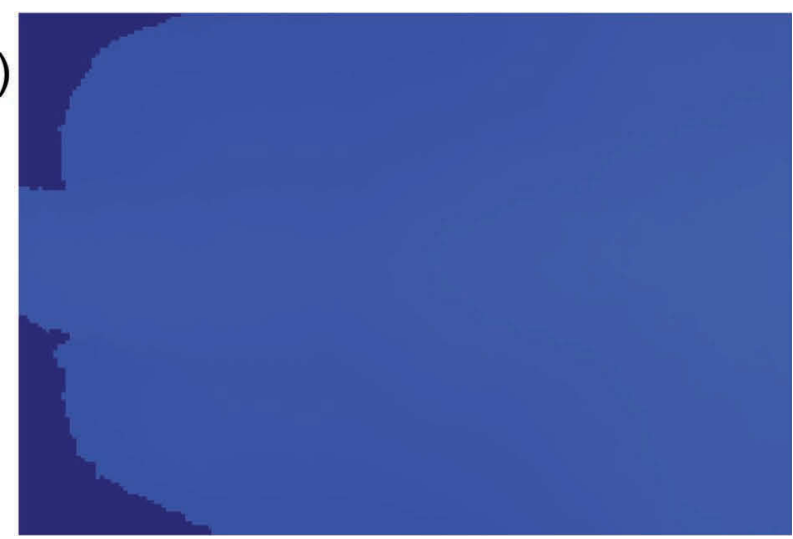

Figure 4. Original image (A) and cropped normalized image (B). Color version available online.

were summed separately, and those with a sum of 0 were removed from the image. To cut the cow's tail out of the image, the indexes of the object's edges in every column were subtracted to calculate the width of the object in every column. The image was cut from the highest indexed column with a width of less than 50 . After image rotation, the image was cropped again, and the left 200 columns were selected first and all others removed; if the image was less than 200 columns, the extra empty columns were attached to the right side of the image. For images with less than 150 rows, extra empty rows were attached to both sides of the image, and for images with more than 150 rows, the mean center of the columns was detected and the image cropped to have 150 rows around or as close as possible to the center. Figure 4 includes 2 images: an original image and the cropped normalized image.

Image Rotation. The angles of the cows in each image were automatically detected by 2 methods; the image was rotated according to both angles and the rotated image with the highest symmetry was selected automatically. In the first method, the angle of rotation was determined using the arctangent of the division of the calculated difference in the row index for the maximum height (the spine) in the front and rear of the cow and the image width. The second method used the angle between the $\mathrm{x}$-axis and the linear regression line of the highest points in every column representing the cow's spine. After each method, a range of $3^{\circ}$ in every direction was tested to correct for minor mistakes; the test was performed by rotating the image at an angle of 0.1 until a $3^{\circ}$ range was reached and calculating the symmetry at each rotation. The symmetry of the image was calculated by subtracting the top part of the image from the bottom part and summing the difference. The symmetry was calculated on a $150 \times 200$ pixel image. Figure 5 shows an example of an image after this step in the algorithm.
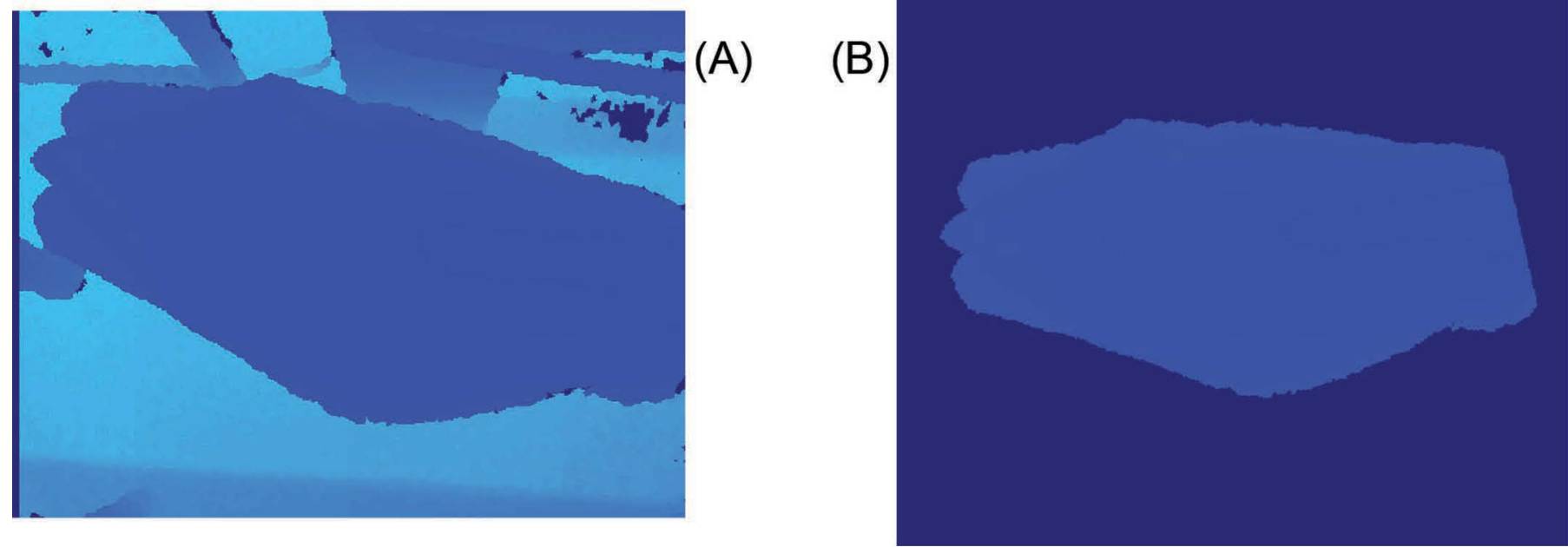

Figure 5. Original image (A) and rotated object (B). Color version available online. 
Image Normalization. The minimum value of the image, set by the threshold at the beginning of the algorithm, was subtracted from all image values; the values were then divided by the maximum value of the image. All pixel values in the image were between the minimum value 0 and maximum value 1 .

Feature Extraction. The $150 \times 200$ pixel images extracted from the movie with a focus on the cow's back end were reshaped as vectors with 30,000 values. The correlation of each point in the vector and the BCS was tested and 6 zones in the image were found to have higher correlations. From each of these zones, 10 pixels with the highest correlation were chosen and their mean was calculated. The 6 mean pixel values found using the correlated zones served as features in the prediction algorithm, and their positions were saved to automatically extract their values from new incoming images (e.g., the test data).

Several features from all pixel values were used in the prediction algorithm. The average relative height, computed as the image-averaged value, showed a dominance of higher or lower values, suggesting a convex or concave 3D curvature, respectively. The relative height standard deviation, computed as the standard deviation of all image values, was also linked to the 3D curvature of the cow. The maximum height of the cow's back end, the same value that was used to normalize the output image of the image processing algorithm, provided a direct connection to the original image value scale, which was suggestive of the size of the cow and its BCS. The number of local minima and maxima of the relative heights in the image, which help the prediction algorithm consider the level of torsion in the cow's back, were a large part of the manual BCS assessment. The average of the Euclidean distances to the cows' outlines from the middle of the far right column was used to learn about the two-dimensional curvature. All features were automatically extracted from each selected frame using the image-processing algorithms described above.

\section{RESULTS}

\section{Automatic Operation}

The system was run automatically, with no human involvement in either guiding the cow traffic or the computer vision process. The automatic movie selection resulted in $100 \%$ of the selected movies having a valid cow back end in the camera's field of view; $100 \%$ of the movies with no cow back end in the camera's field of view were rejected. The rejected movies had obstructions, such as 2 cows touching each other or a following cow's head hiding the tailhead area $(7.82 \%$,
33 movies), or the cow under the camera, rapid movement, or slippage that resulted in not enough sharp frames in the field of view $(2 \%)$.

\section{Accuracy}

The new features used in this research and their correlation to manual BCS are listed in Table 1. The contribution of each separate feature was not high, but the summed accuracy of the entire system was coefficient of determination $=0.75, \mathrm{MAE}=0.26 \mathrm{BCS}$ (one-quarter of a BCS point is known to be the general accuracy of a well-trained expert), and median absolute error = 0.19 . Seventy-four percent of the values appeared to be within a quarter-point distance of the gold reference, $91 \%$ were within a half point, and all $100 \%$ of the assessments were within 1 point from the gold reference. This suggests that, for practical applications, the entire system performance did not fall behind the common performance of human observations.

\section{Sensitivity Analyses}

The influence of the size of the training set and content (K-fold cross-validation) on the model can be seen in Tables 3 and 4 . The size of the training set had little influence on the results. When the training set size was 5,912 ( $50 \%$ of the original training set size), the coefficient of determination and MAE were 0.74 and 0.26 , with a respective decrease and increase of only 0.013 and 0.007 , respectively, compared with the results of models trained with the full training set; even at only $10 \%$ of the training data size, the coefficient of determination was still above 0.7 and the MAE was still below 0.3 . When only 724 images were used in the training set (5\% of the original training set size) the results were far less impressive, with a coefficient of determination of 0.60 and a MAE of 0.37 .

The data for the K-fold cross-validation were randomly divided into K groups, and each time the training was performed with the extraction of 1 group. In the cross-validation, we saw average results of coefficient of determination $=0.67$ and $\mathrm{MAE}=0.34$ (the worst choice of $\mathrm{K}$ ); these results were, as expected, lower than

Table 3. Influence of training set size on $\mathrm{R}^{2}$ and mean absolute error (MAE) for testing data $(100 \%=11,824$ images)

\begin{tabular}{lcc}
\hline Size & Average $\mathrm{R}^{2}$ & Average MAE \\
\hline $100 \%$ & 0.75 & 0.26 \\
$\sim 50 \%$ & 0.74 & 0.27 \\
$\sim 10 \%$ & 0.73 & 0.29 \\
$\sim 5 \%$ & 0.60 & 0.37 \\
$\sim 3 \%$ & 0.50 & 0.39 \\
\hline
\end{tabular}


Table 4. K-fold cross-validation of the model

\begin{tabular}{ccccc}
\hline $\mathrm{K}$ & $\begin{array}{c}\text { Average } \\
\mathrm{R}^{2}\end{array}$ & $\begin{array}{c}\text { Maximum } \\
\mathrm{R}^{2}\end{array}$ & $\begin{array}{c}\text { Average mean } \\
\text { absolute error }\end{array}$ & $\begin{array}{c}\text { Minimum mean } \\
\text { absolute error }\end{array}$ \\
\hline 10 & 0.67 & 0.84 & 0.34 & 0.29 \\
20 & 0.68 & 0.94 & 0.34 & 0.18 \\
30 & 0.67 & 0.95 & 0.34 & 0.14 \\
\hline
\end{tabular}

the model's ability. The analysis showed that even the poorest results had an average MAE $<0.34$ and an average coefficient of determination $>0.67$.

The cross-validation results suggested that the content of the training set is important. The importance of the training data can be seen in 2 places; the first is the large difference between the average measure and the maximum or minimum measure, and the second is the low average measure results. This was expected, even more so due to the inability to ensure that every training set has all BCS groups. The maximum and minimum results were better than the initial ones, indicating that the training data in the research were not specific.

Based on the sensitivity analyses, the training set size should be more than 30 movies and it should include all 5 BCS classes. The number 30 was calculated as $10 \%$ of 294 movies in the training set, and the conclusion that all BCS groups should be in the training set stemmed from the gap in average scores.

\section{Noise Analysis}

Table 5 summarizes the results of the noise analysis. The prediction algorithm showed a low sensitivity to noise, with the ability to cope with a noise ratio of up to 50:1. The results for a noise ratio of 100:1 were very similar to those with no noise - a MAE of 0.26 compared with 0.26 , and an coefficient of determination of 0.7531 compared with 0.753 . The result of added noise at a ratio of 50:1 was a MAE of 0.41 and a coefficient of determination of 0.48 . It should be noted that the added noise was in addition to the natural noise in the data collected from a working dairy farm.

Table 5. Prediction results of the model with added noise

\begin{tabular}{lcc}
\hline $\begin{array}{l}\text { Gaussian noise ratio of } \\
\text { signal to noise }\end{array}$ & Mean absolute error & $\mathrm{R}^{2}$ \\
\hline No noise added & 0.26 & 0.75 \\
$200: 1$ & 0.26 & 0.75 \\
$70: 1$ & 0.26 & 0.75 \\
$60: 1$ & 0.29 & 0.70 \\
$50: 1$ & 0.41 & 0.48 \\
$40: 1$ & 1.16 & -3.29 \\
$20: 1$ & 10.3 & -348.5 \\
\hline
\end{tabular}

\section{DISCUSSION}

\section{Comparison to Previous Research}

Movies and images were captured, selected, and processed automatically. In previously reported studies (Coffey et al., 2003; Bewley et al., 2008; Halachmi et al., 2008; Azzaro et al., 2011; Bercovich et al., 2013) images were partly selected or processed manually. The algorithm presented here (threshold 1, 1,800 mm) achieved background independence; that is, pixel values that were further away from the camera were ignored. This threshold overcame the dependency on image background described in previous studies (Bercovich et al., 2013; Van Hertem et al., 2013, 2014; Viazzi et al., 2014; Caccamo et al., 2015; Shelley et al., 2016) that reported an inability to deal with different backgrounds and also to filter out farm dogs from the movies. Offthe-shelf, low-cost equipment was used (Kinect camera) as opposed to past studies that used expensive equipment [Nikon (Tokyo, Japan) D7000 DSLR camera (Bercovich et al., 2013) and InfraCAM (Flir Systems, West Lafayette, IN) SD thermal camera (Halachmi et al., 2008, 2013)], enhancing this system's commercial applicability.

In addition to automating the whole process, the current research improved measures of performance compared with previous research. In our study, the correlation between manual and automatic BCS in the testing set was 0.76 , as compared with a correlation of 0.31 in the work of Halachmi et al. (2008), who evaluated BCS according to differences between a parabolic estimation of the cow's contour and its actual contour. The methods were improved and retested in Halachmi et al. (2013), and they showed a Pearson correlation coefficient of 0.94 for the training data, which can be closely compared with the root of the coefficient of determination achieved in this research $(0.87$ for the testing set). In previous studies, MAE of 0.34 (Bercovich et al., 2013) when using Fourier descriptors to describe the contour and 0.31 (Azzaro et al., 2011) when using a unified coordinate system to evaluate BCS were found, which is compared with 0.28 found in the testing set in the current research. Our research was calibrated on one database and tested on another database, as opposed to other studies (Halachmi et al., 2008; Azzaro et al., 2011) in which testing was performed only once, strengthening the validity of our results.

\section{Toward Commercial Application}

Local farm conditions, such as camera height, background lighting, ceiling height, and cow width will all need to be considered when installing the automated 
BCS system in other farms. Further research should investigate the influence of other farms' local conditions on the image-processing parameters (e.g., cleanliness, a farmer decision-making frequency, and light conditions: closed barn vs. open cowshed).

The practical application of BCS aims to achieve a balance between economic feeding and good production. Frequent and accurate BCS could help to ensure that the cow is in the correct condition for each stage of her annual cycle and that appropriate dietary changes can be made to correct deficiencies. The proposed camera-based BCS monitoring has 3 contributions: saving labor (replacing the humans that today evaluate BCS manually) as well as providing frequent and objective measurements. Further research should quantify the economics of managing a farm with the proposed automatic BCS.

Our study made use of the first version of the Kinect camera with its specific resolution and sensitivity. A new camera version might require adaptation to the image processing parameters. The algorithm was programmed using Matlab. OpenCV and $\mathrm{C}++$ will run the model faster, with less central processing unit and memory usage for any commercial application.

\section{CONCLUSIONS}

In this study, 14 new features $(6$ relative height features +6 features calculated from image data +2 prior data features) were found to be correlated with BCS. These features seem to be measurable by low-cost 3D camera on a commercial farm. An automated 3D-acquisition cow-walk-through system for BCS was designed and built on farm and located at the milking parlor exit. The system was run automatically, with no human involvement in either guiding the cow traffic or the computer vision process. The system was found to be reliable after several days of unsupervised data acquisition. The system appeared to be robust under commercial farm conditions, added Gaussian noise, and varied sizes of the training data set. The image selection in the image processing algorithm produced $0 \%$ false positives and only $7.82 \%$ misses in selecting movies that included cows' back ends. The system showed improved objectivity over current methods, and it gave results that were close to those of a trained expert. System accuracy was $\mathrm{MAE}=0.26$, coefficient of determination $=0.75$, and a good error rate, with $94 \%$ of the errors under 0.75 and all errors under 1 . The advantages of this system over those in previous studies include automatic image capture and selection as well as low-cost imaging using a commercial, off-the-shelf 3D Kinect camera. Further research should focus on (1) testing several farms and (2) obtaining reference values (manual BCS) from more than 1 expert; reference values from a group of experts will provide a better understanding of sources of errors.

\section{ACKNOWLEDGMENTS}

The authors acknowledge the accurate cow body condition scoring of Uzi Moallem (ARO, Animal Science Institute). Thanks to Aaron Antler of the ARO for building the prototype at the Volcani Center's dairy farm. The authors acknowledge Ephraim Maltz and Victor Alchanatis of the ARO for providing useful comments throughout the project. This research was partially supported by the Ben-Gurion University of the Negev Rabbi W. Gunther Plaut Chair in Manufacturing Engineering, the Israeli Ministry of Agriculture Chief Scientist fund (Rishon LeZion, Israel), project number ARO-459-4369, and the Institute of Agricultural Engineering, ARO.

\section{REFERENCES}

Azzaro, G., M. Caccamo, J. D. Ferguson, S. Battiato, G. M. Farinella, G. C. Guarnera, G. Puglisi, R. Petriglieri, and G. Licitra. 2011. Objective estimation of body condition score by modeling cow body shape from digital images. J. Dairy Sci. 94:2126-2137.

Bercovich, A., Y. Edan, V. Alchanatis, U. Moallem, Y. Parmet, H. Honig, E. Maltz, A. Antler, and I. Halachmi. 2013. Development of an automatic cow body condition scoring using body shape signature and Fourier descriptors. J. Dairy Sci. 96:8047-8059.

Bewley, J. M., A. M. Peacock, O. Lewis, R. E. Boyce, D. J. Roberts, M. P. Coffey, S. J. Kenyon, and M. M. Schutz. 2008. Potential for estimation of body condition scores in dairy cattle from digital images. J. Dairy Sci. 91:3439-3453.

Caccamo, M., G. C. Guarnera, G. Licitra, R. Azzaro, G. Petriglieri, and G. Gallo. 2015. Estimation of cow's body condition score through statistical shape analysis and regression machines from images acquired using low-cost digital cameras. Pages 66-73 in Proceedings of the European Conference on Precision Livestock Farming. Milan Univ., Milan, Italy. Microtome Publishing, Brookline, MA.

Coffey, M. P., T. B. Mottram, and N. McFarlane. 2003. A feasibility study on the automatic recording of condition score in dairy cows. Page 131 in Proc. Ann. Mtg. British Assoc. Anim. Sci. British Assoc. Anim. Sci., Penicuik. Midlothian, UK.

Cyganek, B., and J. P. Siebert. 2011. An Introduction to 3D Computer Vision Techniques and Algorithms. John Wiley \& Sons, New York, NY.

DeLaval. 2015, DeLaval BCS system in a milking robot. Accessed September 2015. http://www.delaval.com/Global/About\%20 DeLaval/Body\%20condition\%20scoring_Brochure_V6_brochure. pdf.

Edmondson, A. J., I. J. Lean, L. D. Weaver, T. Farver, and G. Webster. 1989. A body condition scoring chart for Holstein cows. J. Dairy Sci. 72:68-78.

Ferguson, J. D. 1996. Implementation of a body condition scoring program in dairy herds. Feeding and managing the transition cow. Page 5 in Proceedings of the Penn Annual Conference, University of Pennsylvania, Kennett Square, PA. Center for Animal Health and Productivity, School of Veterinary Medicine, Kennett Square, $\mathrm{PA}$.

Ferguson, J. D., G. Azzaro, and G. Licitra. 2006. Body condition assessment using digital images. J. Dairy Sci. 89:3833-3841.

Ferguson, J. D., D. T. Galligan, and N. Thomsen. 1994. Principal descriptors of body condition score in holstein cows. J. Dairy Sci. 77:2695-2703. 
Hady, P. J., J. J. Domecq, and J. B. Kaneene. 1994. Frequency and precision of body condition scoring in dairy cattle. J. Dairy Sci. $77: 1543-1547$.

Halachmi, I., M. Klopcic, and P. Polak. 2008. Body condition scoring using thermal camera. Page 26 in Proceedings of The 20th Conference on Dairy Science. The Israeli Cattle Breeding Association (ICBA), Jerusalem, Israel.

Halachmi, I., M. Klopčič, P. Polak, D. J. Roberts, and J. M. Bewley. 2013. Automatic assessment of dairy cattle body condition score using thermal imaging. Comput. Electron. Agric. 99:35-40.

Otsu, N. 1975. A threshold selection method from gray-level histograms. IEEE Trans. Syst. Man Cybern. 9:62-66. http://dx.doi. org/10.1109/TSMC.1979.4310076.

Peacock, A. M., J. M. Bewley, and O. Lewis, inventors. 2006. Method and apparatus for the automatic grading of condition of livestock. Patent NZ570714, US 2009/074253 and EP 2027770. IceRobotics Ltd., assignee.

Salau, J., J. Haas, W. Junge, U. Bauer, J. Harms, and S. Bieletzki. 2014. Feasibility of automated body trait determination using the SR4K Time-Of-Flight Camera in cow barns. Springerplus 3:225.

Sharony, D., inventor. 2003. Imaging system and method for body condition evaluation. EU Pat. No 1537531. Filing date: Jul. 27, 2003 (Israel). Publication date: Jun. 8, 2005. Vet-Tech Ltd., assignee.

Shelley, A. N., D. L. Lau, A. E. Stone, and J. M. Bewley. 2016. Short communication: Measuring feed volume and weight by machine vision. J. Dairy Sci. 99:386-391.

Shirai, Y. 2012. Three-Dimensional Computer Vision. Springer Science \& Business Media, Berlin, Germany.

Tedín, R., J. Becerra, and R. Duro. 2014. Building the "Automatic Body Condition Assessment System" (ABiCA), an automatic body condition scoring system using active shape models and machine learning. Pages 145-168 in Recent Advances in Knowledge-based Paradigms and Applications. Vol. 234. J. W. Tweedale and L. C. Jain, ed. Springer International Publishing, Cham, Switzerland.

Van Hertem, T., V. Alchanatis, A. Antler, E. Maltz, I. Halachmi, A. Schlageter-Tello, C. Lokhorst, S. Viazzi, C. E. B. Romanini, A. Pluk, C. Bahr, and D. Berckmans. 2013. Comparison of segmentation algorithms for cow contour extraction from natural barn background in side view images. Comput. Electron. Agric. 91:65-74.

Van Hertem, T., S. Viazzi, M. Steensels, E. Maltz, A. Antler, V. Alchanatis, A. A. Schlageter-Tello, K. Lokhorst, E. C. B. Romanini, C. Bahr, D. Berckmans, and I. Halachmi. 2014. Automatic lameness detection based on consecutive 3D-video recordings. Biosyst. Eng. 119:108-116.

Viazzi, S., C. Bahr, T. Van Hertem, A. Schlageter-Tello, C. E. B. Romanini, I. Halachmi, C. Lokhorst, and D. Berckmans. 2014. Comparison of a three-dimensional and two-dimensional camera system for automated measurement of back posture in dairy cows. Comput. Electron. Agric. 100:139-147.

Weber, A., J. Salau, J. H. Haas, W. Junge, U. Bauer, J. Harms, O. Suhr, K. Schönrock, H. Rothfuß, and S. Bieletzki. 2014. Estimation of backfat thickness using extracted traits from an automatic 3D optical system in lactating Holstein-Friesian cows. Livest. Sci. 165:129-137.

Wildman, E., G. Jones, P. Wagner, R. Boman, H. Troutt, and T. Lesch. 1982. A dairy cow body condition scoring system and its relationship to selected production characteristics. J. Dairy Sci 65:495-501. 
J. Dairy Sci. 99:7724-7724

http://dx.doi.org/10.3168/jds.2016-99-9-7724

(C) American Dairy Science Association ${ }^{\circledR}, 2016$.

\title{
Corrigendum to "Microbiological quality of pasteurized milk on expiration date in Tehran, Iran" (J. Dairy Sci. 99:1796-1801)
}

\author{
Mohammadreza Koushki, Paliz Koohy-Kamaly, Mohammad Azizkhani, and Noushin Hadinia
}

On page 1798 (corrections in bold) in Table 2, the correct percentage for samples with TMC count of 8-9 log $\mathrm{cfu} / \mathrm{mL}$ is $\mathbf{9 . 5 \%}$. The first line of the third paragraph should read "In the present study, the TMC in $\mathbf{4 6 \%}$ of the pasteurized milk samples exceeded $10^{6}(6 \mathrm{log}) \mathrm{cfu} / \mathrm{mL}$, the limit set by INS (ISIRI, 2008) for the total microbial count of raw milk (Table 2).

On page 1800, the Conclusions should read "In the present study, 36.6\% of the samples were in accordance with the Iranian National Standard limits. The mean of total microbial count exceeded the limit, and $\mathbf{4 6 \%}$ of the samples had high total microbial count $\left[>10^{6}(6 \mathrm{log}) \mathrm{cfu} / \mathrm{mL}\right]$. Although the mean of coliform count was above the INS limit, $\mathbf{7 5 . 6 \%}$ of samples had coliform counts in compliance with the INS limit. Only $8.7 \%$ of our samples were contaminated with $E$. coli. It is necessary to improve pasteurized milk quality and address factors influencing the quality of milk, such as the cold chain, in Iran."

The authors regret the errors.

\section{REFERENCES}

Koushki, M., P. Koohy-Kamaly, M. Azizkhani, and N. Hadinia. 2016. Microbiological quality of pasteurized milk on expiration date in Tehran, Iran. J. Dairy Sci. 99(3):1796-1801. 
31st ADSA Discover Conference on Food

Animal Agriculture:

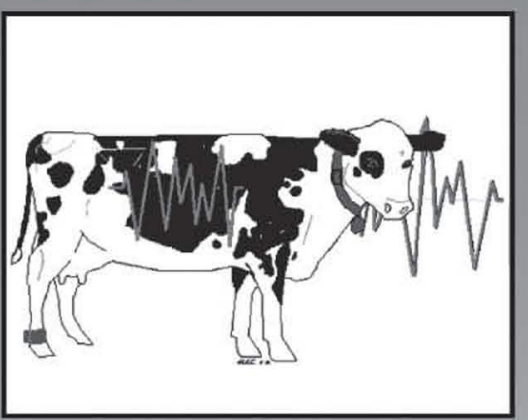

Big Data Dairy Management

November 1-4, 2016

Hilton Chicago/Oak Brook Hills Resort \&

Conference Center in Oak Brook, IL

Hosted by the American Dairy Science

Association"

\section{Conference Format}

The Discover Conference Series is designed to provide a format and venue that encourages in-depth discussion of cuttingedge science.

ADSA Discover Conferences ${ }^{5 M}$ focus on topics of importance to the science of food animal agriculture and are held in a relaxed, informal setting. Sessions for this conference will allow ample time for discussion, networking and relaxation.

\section{Program Committee Organizers:}

Jeffrey Bewley, University of Kentucky (Co-Chair) Christina Petersson-Wolfe, Virginia Tech (Co-

Chair)

Albert De Vries, University of Florida

Alan Fahey, University College Dublin

Miel Hostens, Ghent University

Stephen Leblanc, University of Guelph

Mike Overton, Elanco

Juan M. Tricarico, Innovation Center for U.S. Dairy

American Dairy Science Association Discover Conference Series

Phone: 217/356-5146 Fax: 217/398-4119

Email: adsa-discover@assochq.org

\section{Big Data Dairy Management}

\section{Conference Objective}

Across all industries, the availability of increasingly powerful computers and new technologies provides new business management opportunities. In the last few years, most large companies have embraced the concept of "big data" techniques as part of their management strategy. Definitions of big data vary. But, in general, the term refers to using large data sets for complex decisions where traditional data processing techniques may lack. The key components of big data are analysis, capture, data curation, search, sharing, storage, transfer, visualization, and information privacy. Big data often involves using predictive analytics to analyze existing data sets in new ways. Another key characteristic of big data is merging data from multiple sources into cloud computing. For example, in the dairy industry, big data may involve combining DHI production records, financial records, precision dairy technology data, health records, milk cooperative records, historical weather data, genomic evaluations, ration and feeding management data, and human resource data into one large database. Combining this information helps to improve decision-making, operational efficiency, cost and revenue optimization, and risk management.

The dairy industry remains a perfect application of decision science and big data because: (1) it is characterized by considerable price, weather and biological variation, and uncertainty, (2) technologies, such as those that monitor dairy cow yield, physiology, and behavior are easily available, (3) and the primary output, fluid milk, is difficult to differentiate, increasing the need for alternative means of business differentiation. Big data represents a potential management breakthrough for the dairy industry. Various industry and academic players have been working within this area without a venue to discuss overall strategies and opportunities.

\section{Tentative Conference Themes}

- What is Big Data?

- How is it being used in other industries

- Big data analysis techniques

- Data integration and visualization

- Sensor data use and management

\section{Who Should Attend?}

This program will address issues important to university, government and industry researchers; university extension specialists; software developers, precision dairy technology manufacturers, pharmaceutical companies, genetics providers, consultants, nutritionists, and veterinarians. Graduate students are also invited to attend.

\section{Travel}

The conference will be held at Hilton Chicago/Oak Brook Hills Resort \& Conference Center in Oak Brook, IL, situated on 150 acres west of downtown Chicago and 17 miles from both $\mathrm{O}^{\prime}$ Hare and Midway airports. For the latest travel information, go to the DC31 web page.

\section{Registration \& Hotel Accommodations}

Information regarding registration and hotel accommodations is available on the DC31 web page.

\section{DISCOVER}

For complete conference information, including the latest program and registration information, go to: http://adsa.org/Meetings/DiscoverConferences/31stDiscoverConference.aspx 